\title{
Normativität in der \\ Technikfolgenabschätzung
}

Einleitung in das TATuP-Thema

Linda Nierling, Institut für Technikfolgenabschätzung und Systemanalyse (ITAS), Karlsruher Institut für Technologie (KIT),

Karlstraße 11, 76133 Karlsruhe (linda.nierling@kit.edu), (D orcid.org/0000-0001-9129-550X

Helge Torgersen, Institut für Technikfolgen-Abschätzung, Österreichische Akademie der Wissenschaften

(Helge.Torgersen@oeaw.ac.at), (1) orcid.org/0000-0002-8863-7959

Neutralität galt lange als unhinterfragte Grundlage im Selbstverständnis von Technikfolgenabschätzung (TA). Dieser Fokus verstellte allerdings den Blick darauf, dass normative Aspekte nicht außer Acht gelassen werden dürfen - sei es in den Ergebnissen von TA-Analysen oder in normativen Setzungen, die im TA-Prozess auftreten. Im TATuP-Thema dieses Heftes wird "Normativität in der TA“ auf drei Ebenen adressiert: in der Funktion von TA als Politikberatung, im Kontext des TA-Forschungsprozesses und in der Auseinandersetzung um ihren „normativen Kern“. Angesichts manch autoritärer Tendenzen auch in westlichen Demokratien ist die Debatte um die Rolle von Normativität in der TA heute besonders aktuell.

\section{Normativity in technology assessment}

Introduction to the special topic

Neutrality has long been considered a key prerequisite of technology assessment (TA). The need to stay neutral often obscured the importance of normative aspects of TA - be it in the findings or in normative settings in the TA process. The special topic addresses normativity in TA at three levels: (1) regarding TA's role as policy advisor, (2) in the context of the research process, and (3) with respect to its "normative core". The problem of normativity in TA gains significance in the light of recent authoritarian tendencies also in Western democracies.

Keywords: normativity, values, neutrality, normative core, policy advice

This is an article distributed under the terms of the Creative Commons Attribution License CCBY 4.0 (https://creativecommons.org/licenses/by/4.0/)

https://doi.org/10.14512/tatup.28.1.11
„Die meisten TA-Analytiker fassen nun das Technology Assessment als ein ,normatives Instrument' auf, dessen Aufgabe es ist, plausible - oder auch wünschbare alternative Zukünfte zu entwerfen (Szenarien) und Wege (Optionen, Maßnahmen) zu beschreiben und in Bezug auf Bedingungen und Folgen zu analysieren, auf denen diese ,Zukünfte' erreicht werden können."

(Paschen 1986, S. 35)

Dieses Zitat aus den Anfängen der Technikfolgenabschätzung (TA) im deutschsprachigen Raum zeigt auf, dass das Thema der Normativität, d. h. das Problem der ,Nicht-Trennbarkeit von Fakten und Normen“ (Kollek in diesem Heft, S. 16) von Beginn an in der TA aufgeworfen wurde. Dass dies zunächst nicht theoretisch ausdifferenziert wurde (Böschen und Dewald 2018), lässt sich auch mit der Strategie der in dieser frühen Phase etablierten TA-Einheiten an nationalen Parlamenten erklären. Diese wollten den Anschein von Parteilichkeit vermeiden und so galt weniger das Offenlegen normativer Bezüge, sondern vielmehr Neutralität als Voraussetzung für eine unabhängige Politikberatung. Gut 30 Jahre später ist Neutralität als Mythos der TA entzaubert (Torgersen 2019).

Das Ausblenden der Normativitätsfrage war solange wenig problematisch wie Demokratie, Gewaltenteilung und eine Art sozialverträgliche Technikgestaltung über Parteigrenzen hinweg als selbstverständlich galten. Dieser Konsens erscheint angesichts populistisch-autoritärer Tendenzen in Politik und Gesellschaft heute gefährdet. Für die TA erhebt sich damit die Frage nach den eigenen Werten und Normen: Ist der Bezug auf Neutralität heute noch zeitgemäß? Welche Konsequenzen - Unparteilichkeit bewahren oder Position beziehen - ergeben sich aus Wertekollisionen (Delvenne und Parotte 2019)? Und wie lässt sich Normativität für die Technikfolgenabschätzung fassbar machen?

Die in diesem TATuP-Thema versammelten Beiträge sowie das Interview in diesem Heft zeigen, dass diese Fragen über aktuelle politische Bezüge weit hinausgehen. So werden in der 
TA-Community derzeit normative Bezüge aus verschiedenen Blickwinkeln diskutiert: als theoretische Anforderungen (Böschen und Dewald 2018), als „Politics der TA“ (Hennen und Nierling 2019) oder mit Bezug auf die Praxis (wie auf der TA18 in Wien). Außerdem ist ,die TA “vielgestaltig: In der politischen Beratung aktiv, erarbeitet sie ebenso originäres wissenschaftliches Wissen und formiert sich als inter- und transdisziplinäre Wissenschaftsdisziplin über Ländergrenzen hinweg (Hennen und Nierling 2015; Hahn et al. 2018). Beides bringt Herausforderungen für die Arbeitsweise und Themenwahl sowie für die Selbstverortung von TA-Forscherinnen und -Forschern im politischen und Innovationssystem und damit für die Wahl normativer Bezugspunkte mit sich (Dobroć et al. 2018; Dusseldorp 2014).

\section{Neutralität ist als Mythos der TA entzaubert.}

Nun ist die Operationalisierung von Normativität für die TA kein einfaches Unterfangen, nicht zuletzt, weil Begriff und Konzept lange Traditionen in Philosophie, Soziologie und Rechtswissenschaft haben. Diese umfassend für die TA aufzubereiten, kann hier nicht Gegenstand sein; eine vielsagende Definition soll aber in Erinnerung gerufen werden. So lässt sich Normativität verstehen als „Bezeichnung für Aussagen, in denen eine Bewertung ausgedrückt wird (z. B. richtig, gut) verbunden mit der Forderung, sich dieser Bewertung anzuschließen“" (Woesler und Lautmann 2011, S. 476). Beide Elemente von Normativität sind für die TA relevant: zum einen die Bewertung von Aussagen, zum anderen der damit verbundene Aufforderungscharakter, diese Bewertung zu teilen. Gerade letzteres ist eine wesentliche Herausforderung für die TA und wohl auch Grund dafür, dass sich Normativität und TA nicht so einfach zusammenbringen lassen.

\section{Beiträge in diesem Heft}

Dieses Verhältnis offen zu legen, epistemische Unterschiede zwischen Fakten und Normen in der TA-Praxis zu thematisieren und das inhärent Normative der TA überhaupt diskutierbar zu machen, ist Anliegen dieses TATuP-Themas. Die versammelten Beiträge adressieren dabei drei Ebenen: (1) die TA in ihrer beratenden Funktion, (2) die Reflexion ihrer wissenschaftlichen Praxis und (3) ihre Selbstverständigung.

\section{Drei Ebenen der Normativität}

Die erste Ebene betrifft die Funktion der TA als Instrument der Politik- bzw. Gesellschaftsberatung. Die Forderung nach Neutralität wurde bereits im Gründungsdokument des Office of Technology Assessment von 1972 festgeschrieben als ,providing neutral, competent assessments about the probable beneficial and harmful effects of new technologies" (Bimber 1996, S. 26). Der Neutralitätsanspruch war Voraussetzung, um TA im politischen Kontext zu installieren - auch wenn dies letztlich von der geschickten Positionierung zwischen ,hostile positions from relevant players in the innovation system and a defence against accusations of allegedly following a hidden agenda of ,technology arrestment ““ (Hennen und Nierling 2015, S. 7) abhängig war.

Die Praxis gestaltete sich mitunter schwierig. Das OTA erprobte unterschiedliche Neutralitätsstrategien (Bimber 1996), etwa eine Orientierung an gemeinwohlorientierter „besserer“ Politik, die aber als Präferenz für die Demokratische Partei ausgelegt wurde. Die folgende Phase strikter Neutralität führte zu realitätsfernen Berichten und Bedeutungsverlust. Erfolgreicher war man mit einer Gleichgewichtspolitik, die konkurrierende Interessen gemäß dem Prinzip ,something for everybody“ bediente. Ein Nachhall dieser Differenzen lässt sich noch heute an verschiedenen Parlamenten beobachten: So enthält sich etwa das britische Parliamentary Office of Science and Technology (POST) jeglicher Stellungnahme, während der dänische Technologierat eigenständige Empfehlungen gab (Nentwich 2016). Ob TA in der Praxis normative Positionen vertritt, ist also keine ontologische Frage, vielmehr geht es um die strategische Verbindung von Analyse und Bewertung in den jeweiligen spezifischen Kontexten von TA.

Wie die Beiträge in diesem Heft deutlich machen, ist das Parlament jedoch nicht der einzige Adressat, auch wenn parlamentarische TA für die historische Entwicklung wichtig war. So zeigen Kastenhofer et al., dass Öffentlichkeit, Verwaltung und viele andere gesellschaftliche Akteure unterschiedliche, auch normative Anforderungen an TA stellen (siehe dazu auch Ganzevles et al. 2014). Allerdings ziehen Beziehungen zu den Auftraggeberinnen und Auftraggebern bzw. Adressatinnen und Adressaten von TA-Studien Kastenhofer et al. zufolge keine direkten Abhängigkeiten nach sich. Angesichts der dabei entstehenden Spannungsfelder plädiert Kollek für einen Ersatz (fiktiver) Neutralität durch ,reflexive“ Normativität, d.h. das ständige Bewusstmachen normativer Bezüge, unter denen TA durchgeführt wird, um die oft unbewussten Selektivitäten im TA-Prozess zu vermeiden (siehe auch das Konzept des „doing reflexivitiy“ in Bauer und Kastenhofer 2019). Diese Selektivitäten können sowohl die Inhalte der Beratung als auch deren konzeptionelle Grundlage betreffen - Lucivero et al. unterscheiden daher zwischen einer ,in-normativity“, die sich aus den Werten und Normen der Teilnehmerinnen und Teilnehmer am TA-Prozess und denen der durchführenden Praktikerinnen und Praktikern speist, und einer „,meta-normativity“, mit der die grundlegenden inhärenten Ziele der TA gemeint sind. Einen Vorschlag für die praktische Umsetzung der Forderungen nach normativer Transparenz auf der Basis eines moralpragmatischen Ansatzes liefert Weydner-Volkmann mit dem Konzept der moralischen Landkarten, mit deren Hilfe sich die angewandte Ethik in den TA-Prozess integrieren und für TA-Analysen operationalisieren lässt.

Die zweite Ebene betrifft den Umgang mit offenen oder verdeckten normativen Setzungen im TA-Forschungsprozess. Wie 
in anderen wissenschaftlichen Disziplinen gibt es diesbezüglich auch in der TA viele Beispiele (Dieckhoff 2015). Mit dem Konzept der ,in-normativity“ gelingt es Lucivero et al. in diesem Heft, Annahmen und Werturteile in unterschiedlichen Phasen zu thematisieren. In diesem Sinne untersuchen auch Böschen et al. den Umgang mit Indikatoren im Forschungsprozess am Beispiel der Nachhaltigkeitsbewertung. Sie unterscheiden, ob die Umgebung eines Nachhaltigkeitsproblems ,kontextoffen“ in die Bewertung einfließt oder ob die Indikatoren ,kontextneutralisierend“ nur ein Binnenproblem abbilden. Auf forschungsmethodischer Ebene schlagen Mader et al. ein computergestütztes Verfahren vor, um Werthaltungen von Teilnehmerinnen und Teilnehmern in partizipativen Verfahren transparent zu machen und so normative Ein- der Nachhaltigkeit, soziale[n] Ausgleich und gerechte Verteilung von Risiken und Chancen sowie de[n] Vorrang von gesellschaftlichen Problemen vor rein ökonomischen“.

Ist der normative Kern der TA also mit Gemeinwohl, demokratischen Werten und Verfahren verbunden? Wie stark soll TA Werte und Werthaltungen integrieren und sich auf dieser Basis in gesellschaftliche Diskurse einmischen? Ist der TA gar eine gesellschaftliche „Normenbegründungsfunktion“ (Woopen und Mertz 2014, S. 46) inhärent? Einen Zugang zu der Vielschichtigkeit dieser Fragen bietet das Interview mit Grunwald und Delvenne in diesem Heft. Beide betonen, dass TA seit jeher dem Wertekanon der Aufklärung und einer soliden, gelebten Demokratie verpflichtet sei. Allerdings ziehen sie jeweils unterschied-

\section{Wie stark soll TA Werte und Werthaltungen integrieren und sich auf dieser Basis in gesellschaftliche Diskurse einmischen?}

stellungen für die diskursive Vielfalt nutzen zu können. Während diese Beiträge im Forschungsprozess ansetzen, schlagen Hillerbrand et al. vor, normative Setzungen an dessen Anfang zu stellen. Ihr Capability-Ansatz als Rahmenkonzept für TA-Analysen stellt subjektive Wertsetzungen dezidiert ins Zentrum. Etwa bei der Digitalisierung des Energiesektors kann ein solcher Ansatz normative Bezugspunkte schaffen, um unterrepräsentierte Ansichten offenzulegen und so einen kognitiven Mehrwert für die TA zu erzielen. Diese Beiträge werfen zwar nur Schlaglichter auf den TA-Forschungsprozess, zeigen aber mit großer Deutlichkeit, dass das Ausleuchten und der Umgang mit normativen Elementen einen reflexiven Ansatz erfordern.

Jenseits funktionaler Fragen und des Problems normativer Setzungen im Forschungsprozess fordern die Beiträge auf einer dritten Ebene auch dazu auf, sich über Wertgrundlagen der TA $\mathrm{zu}$ verständigen. Kann es so etwas wie einen normativen Kern geben? Ist angesichts ihrer heutigen Vielgestaltigkeit eine Verständigung über ein ,shared ethos of TA“ (Hennen und Nierling 2019, S. 20) überhaupt möglich? Wie Hennen in diesem Heft zeigt, ist das Problem, ob Werte in der Wissenschaft offen vertreten werden sollten so neu nicht, befasste sich doch bereits Max Weber intensiv mit der wissenschaftlichen Wert(urteils)freiheit. Diese Debatte weist erstaunliche Bezüge zum hier diskutierten Problem auf: Es geht nicht darum, $o b$ Wissenschaft (und damit TA) normativ sei - sie ist es notwendigerweise - , sondern darum, wie man mit dieser Normativität umgehen könne.

Wie lässt sich die Normativität von TA also inhaltlich füllen? Nach Lucivero et al. stellt die ,meta-normativity“ von TA ,the embedded normative ideal that a more pluralistic process will produce better outcomes and benefits for society" dar (S. 23 in diesem Heft). Dahingegen plädiert Grunwald (2019, S. 5) etwas breiter für ,TA's obligation to transparency, inclusion, and democratic debate“ als kleinstem gemeinsamen (normativen) Nenner. Torgersen (2019, S. 26) versteht hierunter die „Werte liche Schlüsse daraus, was mitunter erhebliche Auswirkungen auf die Praxis hat. Nach Grunwald erlaubt die notwendige Inklusion unterschiedlicher Positionen im TA-Prozess keine Präferenz für bestimmte sachliche Lösungen. Delvenne hingegen fokussiert auf die normative Verankerung der TA auch auf der Faktenebene; demnach müsse TA sich zu bestimmten Positionen eindeutig bekennen.

Die theoretische Verständigung auf geteilte Werte der TA und der praktische Umgang damit in Beratung und Forschung bleiben also Gegenstand der Auseinandersetzung. Die hier versammelten Beiträge liefern wesentliche Anknüpfungspunkte, sowohl in Bezug auf das Selbstverständnis der TA und den eigenen Umgang mit Werten als auch in Bezug auf den Forschungsprozess und angemessene TA-Methoden.

\section{Ausblick}

Die normativen Bezüge der TA sind also vielschichtig. Dieser Befund steht in Gegensatz zur verbreiteten Annahme eines inhärenten ,normativen Defizits“ (Grunwald 1999) der TA, das vielfach Anlass zu Kritik gab. Insbesondere im Vergleich mit späteren Ansätzen wie Responsible Research and Innovation (RRI) wurde der TA zuweilen normative Blindheit vorgeworfen (van Lente et al. 2017) bzw. RRI als moralischer Gegenentwurf positioniert (Fisher 2017). Inzwischen liegt die ambivalente Position der TA zwischen Neutralitätsanspruch und normativen Bezügen offen und es werden unterschiedliche Ansätze des Umgangs damit vorgeschlagen. Die Verständigung über geteilte - auch theoriebasierte - Wertbezüge und daraus abzuleitende Verpflichtungen im Umgang mit gesellschaftlichen Problemen steht freilich noch am Anfang.

In der weiteren Auseinandersetzung um die Normativität in der TA sind (mindestens) zwei Dimensionen zu beachten: Einer- 
seits muss der TA-Prozess den Ansprüchen an die Wissenschaftlichkeit und die Ausgewogenheit der eingebrachten Perspektiven genügen. Das bedeutet, Fakten zusammen zu tragen und ihre Relevanz nach wissenschaftlichen Kriterien zu beurteilen, um Unterscheidungen zu treffen. Andererseits sind differente Interessen und Sichtweisen bzw. Weltanschauungen unterschiedlicher Akteure zu integrieren. TA liefert ja nicht nur konkrete Ergebnisse, sondern lässt sich als Teil eines (gesellschaftlichen) Bewusstwerdungsprozesses verstehen, in dessen Verlauf technische Zukünfte entworfen und alternative Lösungen definiert werden. Dabei erscheinen gewisse normative Setzungen unausweichlich. Diese zu verhandeln, anzupassen und Verfahren des reflexiven Umgangs damit zu entwickeln bleibt zentrale Herausforderung für die TA-Community.

\section{Literatur}

Bauer, Anja; Kastenhofer, Karen (2019): Policy advice in technology assessment. Shifting roles, principles and boundaries. In: Technological Forecasting and Social Change 139, S. 32-41.

Bimber, Bruce (1996): The politics of expertise in congress. The rise and fall of the Office of Technology Assessment. New York: State University of New York Press.

Böschen, Stefan; Dewald, Ulrich (2018): Theorie der Technikfolgenabschätzung reloaded. Ten years after. In: TATUP - Zeitschrift für Technikfolgenabschätzung in Theorie und Praxis 27 (1), S. 11-13. D0I: 10.14512/tatup.27.1.11.

Delvenne, Pierre; Parotte, Celine (2019): Breaking the myth of neutrality. Technology assessment has politics, technology assessment as politics. In: Technological Forecasting and Social Change 139, S. 64-72.

Dieckhoff, Christian (2015): Modellierte Zukunft. Energieszenarien in der wissenschaftlichen Politikberatung. Bielefeld: transcript.

Dobroć, Paulina; Krings, Bettina-Johanna; Schneider, Christoph; Wulf, Nele (2018): Alternativen als Programm. Plädoyer für einen Perspektivenwechsel in der Technikfolgenabschätzung. In: TATuP - Zeitschrift für Technikfolgenabschätzung in Theorie und Praxis 27 (1), S. 28-33. D0I: 10.14512/tatup.271.28. Dusseldorp, Marc (2014): Technikfolgenabschätzung zwischen Neutralität und Bewertung. In: APuZ 6-7, S. 25-30

Fisher, Eric (2017): Entangled futures and responsibilities in technology assessment. In: Journal of Responsible Innovation 4 (2), S. 83-84.

Ganzevles, Jürgen; van Est, Rinie; Nentwich, Michael (2014): Embracing variety. Introducing the inclusive modelling of (parliamentary) technology assessment. In: Journal of Responsible Innovation 1 (3), S. 292-313.

Grunwald, Armin (1999): Technology assessment or ethics of technology? Reflections on technology development between social sciences and philosophy. In: Ethical Perspectives 6 (2), S. 170-182.

Grunwald, Armin (2019): Technology assessment in practice and theory. New York: Routledge.

Hahn, Julia; Ladikas, Miltos; Scherz, Constanze (2018): Think global! Reflections on a global technology assessment. In: Michael Decker, Ralf Lindner, Stephan Lingner, Constanze Scherz und Mahshid Sotoudeh (Hg.): „Grand Challenges“ meistern. Der Beitrag der Technikfolgenabschätzung. Baden-Baden: Nomos, S. 41-49.

Hennen, Leonhard; Nierling, Linda (2015): Taking stock of TA in Europe and abroad. Introduction to the thematic focus. In: TATuP - Zeitschrift für Technikfolgenabschätzung in Theorie und Praxis 24 (1), S. 4-10.

Hennen, Leonhard; Nierling, Linda (2019): The politics of technology assessment. Introduction to the special issue. In: Technological Forecasting and Social Change 139, S. 17-22.

Nentwich, Michael (2016): Parliamentary technology assessment institutions and practices. A systematic comparison of 15 members of the EPTA network. ITA-manu:script 16-02. Vienna: ITA/OEAW.

Paschen, Herbert (1986): Technology Assessment. Ein strategisches Rahmenkonzept für die Bewertung von Technologien. Technik und Parlament. In: Meinolf Dierkes, Thomas Petermann und Volker von Thienen (Hg.) Technikfolgenabschätzung. Konzepte, Erfahrungen, Chancen. Berlin: edition sigma, S. 21-46.

Torgersen, Helge (2018): Die verborgene vierte Dimension. Normative Reflexion als Erweiterung der Theorie der Technikfolgenabschätzung. In: TATuP Zeitschrift für Technikfolgenabschätzung in Theorie und Praxis 27 (1), S. 21-27. DOI: 10.14512/tatup.27.1.21.

Torgersen, Helge (2019): Three myths of neutrality in TA. How different forms of TA imply different understandings of neutrality. In: Technological Forecasting and Social Change 139, S. 57-63.

van Lente, Harro; Swierstra, Tsalling; Joly, Pierre-Benoit (2017): Responsible innovation as a critique of technology assessment. In: Journal of Responsible Innovation 4 (2), S. 254-261.

Woesler, Christine; Lautmann, Robert (2011): „normativ“. In: Werner FuchsHeinritz et al. (Hg.): Lexikon zur Soziologie. Wiesbaden: VS Verlag für Sozialwissenschaften, S. 476.

Woopen, Christiane; Mertz, Marcel (2014): Ethik in der Technikfolgenabschätzung. In: APuZ 6-7, S. 40-46.
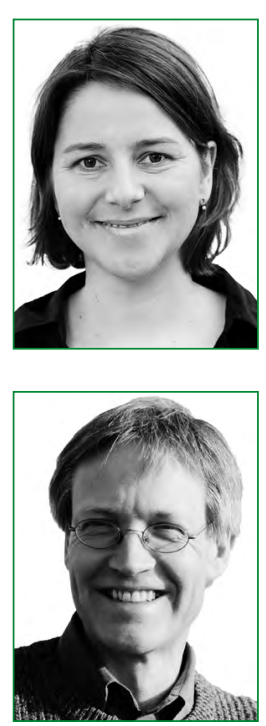

\section{DR. LINDA NIERLING}

arbeitet als Sozialwissenschaftlerin am Institut für Technikfolgenabschätzung und Systemanalyse (ITAS) des Karlsruher Instituts für Technologie (KIT). Ihre Arbeitsschwerpunkte umfassen die Digitalisierung der Arbeitswelt, soziale Aspekte von assistiven Technologien und Konzepte und Methoden von Technikfolgen.

\section{DR. HELGE TORGERSEN}

arbeitet seit 1990 am Institut für TechnikfolgenAbschätzung der Österreichischen Akademie der Wissenschaften, zuvor war er in der molekularbiologischen Forschung an der Universität Wien tätig. Hauptinteressen sind Biotechnologiepolitik, Risikoabschätzung, öffentliche Wahrnehmung und Kommunikation neuer Technologien. 